\title{
Experimental Methods Using Photogrammetric Techniques for Parachute Canopy Shape Measurements
}

\author{
Thomas W. Jones ${ }^{1}$, James M. Downey ${ }^{2}$, and Charles B. Lunsford. ${ }^{3}$ \\ NASA Langley Research Center, Hampton VA, 23681 \\ Kenneth J. Desabrais ${ }^{4}$, and Gregory Noetscher ${ }^{5}$ \\ U.S. Army Natick Soldier Center, Natick MA
}

\begin{abstract}
NASA Langley Research Center in partnership with the U.S. Army Natick Soldier Center has collaborated on the development of a payload instrumentation package to record the physical parameters observed during parachute air drop tests. The instrumentation package records a variety of parameters including canopy shape, suspension line loads, payload 3-axis acceleration, and payload velocity. This report discusses the instrumentation design and development process, as well as the photogrammetric measurement technique used to provide shape measurements. The scaled model tests were conducted in the NASA Glenn Plum Brook Space Propulsion Facility, OH.
\end{abstract}

\section{Introduction}

$\mathrm{P}$ arachute systems play a critical role in many science and military missions. Currently, NASA's exploration program and the U.S. Army's air delivery systems programs are seeking measurement technologies to support experimental and qualification testing of new and modified parachute concepts. Experimental testing to measure the physical parameters involved during the inflation and decent phases of parachutes is a key requirement to obtain a quantitative assessment of the parachutes performance. The objectives of these measurements are to monitor the aerodynamics and structural dynamics of parachutes and to provide a database for the verification and validation of the mathematical/computer models developed to predict the performance of parachute concepts.

Scaled model ground testing of parachute systems have been previously conducted in both wind tunnels and low altitude airdrops. ${ }^{1}$ However, the development activities here are aimed at correlating shape information with other parameters including acceleration, velocity, load, and canopy configurations. The efforts described to acquire shape information of the canopy and the skirt appears to be a first in experimental research where quantitative tools were employed. A detailed knowledge of the parachute canopy shape is important for the design and stress analysis of parachutes. ${ }^{2}$ The testing focused on two canopy sizes, 3.5 and $7 \mathrm{ft}$ diameter. The canopies tested in both size categories had two gore configurations; one with 16 gores the other having only 2. To minimize flow field interference the payload size was restricted to 6 inches in diameter for the larger canopy and 4 inch diameter for the smaller $3.5 \mathrm{ft}$ canopy. The payload instrumentation package was self contained and controlled through radio frequency (RF) signals. The necessary power, control, acquisition and storage components were all located within the package. The measurement signals from the sensors and the on-board video were synchronized through on-board triggering. Visual indicators within the video stream were used as a backup mechanism to verify the synchronization between cameras.

The tests were conducted in the 30m vacuum chamber at the NASA Glenn Space Propulsion Facility (SPF) located at Plum Brook station, $\mathrm{OH}$. The facility has the capability to provide variable pressure and temperature test conditions. The tests described here were conducted at ambient conditions. For this series of tests the most desirable attributes of the chamber were the constant temperature and the elimination of air currents.

\footnotetext{
${ }^{1}$ Electronics Engr, ASOMB, 18 Langley Blvd/ MS 493. AIAA Member.

${ }^{2}$ Electronics Engr, ASOMB, 18 Langley Blvd/ MS 493, AIAA Member.

${ }^{3}$ Electronics Engr, ASOMB, 18 Langley Blvd/ MS 493.

${ }^{4}$ Research Aerospace Engineer, WarPADD, 15 Kansas St./AMSRD-NSC-WP-AT, AIAA member.

${ }^{5}$ Electrical Engineer, JPAD ACTD, 15 Kansas St./AMSRD-NSC-WP-JP.
} 
The tests were conducted using a guide-wire extended from the floor to the ceiling of the chamber. The guidewire passed unimpeded through the payload and the vent of the parachute. To maintain its position the guide-wire was tensioned with a constant $300 \mathrm{lb}$. load to minimize movement from the aerodynamic loads produced by the parachute during the drop.

The graphic shown in Figure 1a. typifies the drop from the center position within the chamber. The accompanying image in Figure 1b. shows the payload and canopy in the pre-drop position prior to being lifted to the top of the chamber. In subsequent sections of this paper a brief background on photogrammetric measurements will be presented, followed by a detailed description of the measurement system components and measurement process.

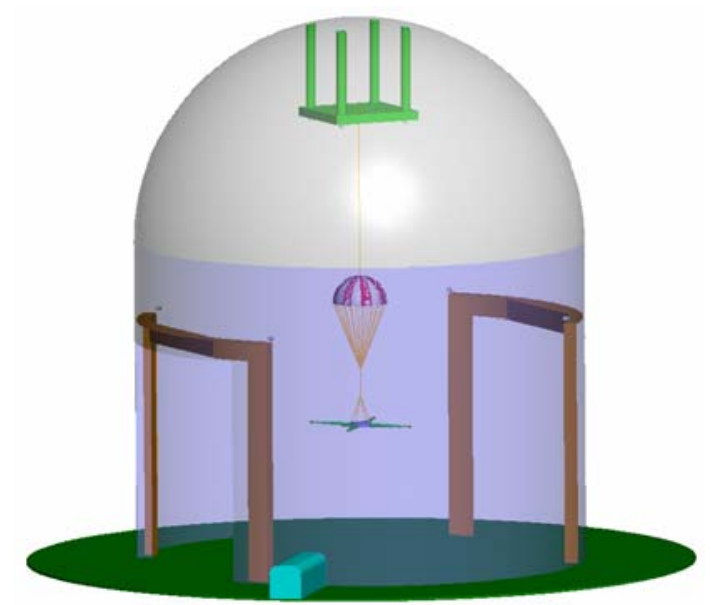

Figure 1a. Controlled drop within the $30 \mathrm{~m}$ chamber at SPF.
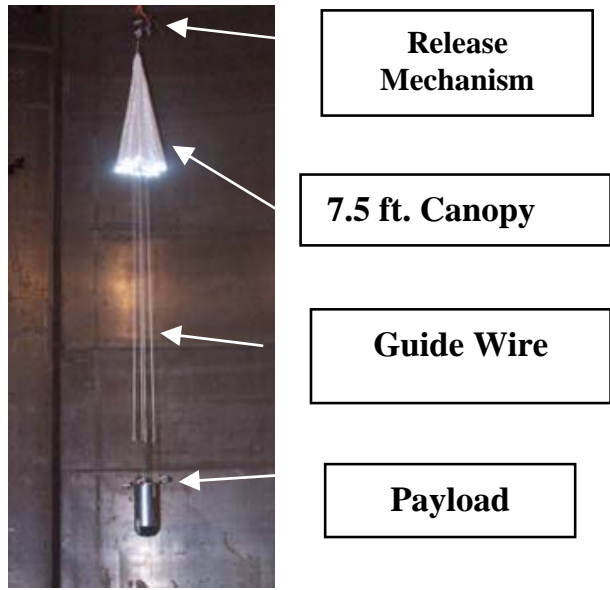

\section{Figure 1b. Parachute with Payload shown in Pre-drop configuration.}

\section{Background: Photogrammetric Measurement}

Historically the shape of the canopy during inflation and descent has been determined qualitatively, however modern video imaging technology has provided a tool capable of supporting such a measurement quantitatively. To provide canopy shape information, NASA Langley Research Center (LaRC) leveraged its success and understanding of optical systems and photogrammetric measurement technologies. Most recently this technology has been applied to the In-Space Propulsion programs $20 \mathrm{~m}$ Solar Sail ground validation test program. ${ }^{3}$ The two programs share many similarities in terms of metrology; their large scale and "membrane like" structure and exhibit non-linear behavior during deployment and inflation. A goal of this effort was the development of a scalable imaging system that supports both ground based (i.e. platform drops, wind tunnels) and high altitude airdrops.

Photogrammetry is the science of measuring the location and size of three-dimensional (3D) objects with photographs. The image analysis procedures are related to point triangulation methods used in surveying. The power of the technique is recognized in its ability to measure hundreds or even thousands of object points simultaneously. Measured sets of object points, also known as "point clouds," can characterize shape. Sequences of images can characterize structural dynamic properties (i.e., vibration) as well. The photogrammetric technique records the object from multiple camera positions and calculates 3D coordinates of discrete points on the object's surface by triangulation of light rays corresponding to the discrete points. 


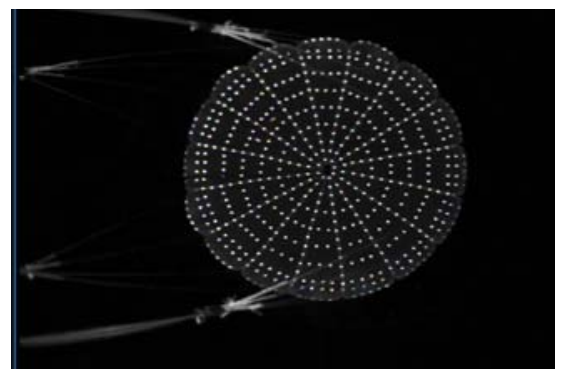

Figure 2. Retroreflective targets on canopy. Payload camera view.
For the test sequence the shape information for the canopy was derived photogrammetrically from a random distribution of discrete points, or targets, on the inside of the canopy that were visible to each of the two on-board cameras. The points were created using retroreflective tape applied to the canopy. The 0.375 -inch diameter target points were constructed from retroreflective tape. A typical recorded image (ref. Figure 2) from the on-board cameras highlights the high contrast created in the imagery with the retroreflective points. The retroreflective material was not porous, but it was considered to have a negligible effect on the overall porosity of the canopy.

To validate the accuracy of the shape solution derived for the on-board cameras, an independent set of digital cameras were positioned on the ground to simultaneously record the canopy during the descent phase. While the limited field of view of the ground-based cameras could not provide shape measurement information over the full range of the descent, they provided enough information to validate the measurements of the on-board system.

\section{Payload Instrumentation and Mechanical Components}

A significant challenge in developing the instrumentation package to meet the test requirements was the volume constraint. The payload provided housing for the instrumentation as well as ballast. The illustration in Figure 3 outlines the basic mechanical design of the payload. The package provided two compartments; one for instrumentation, and the other for the calibrated weights. The 6 inch wide payload provided a modest 5.6 inch high compartment for instrumentation. A similar 5.6 inch compartment was incorporated into the design to accommodate the ballast. A 4 inch payload of similar proportions was designed to operate with the smaller canopies. For the purposes of this report the discussion will be limited to the 6 inch payload.

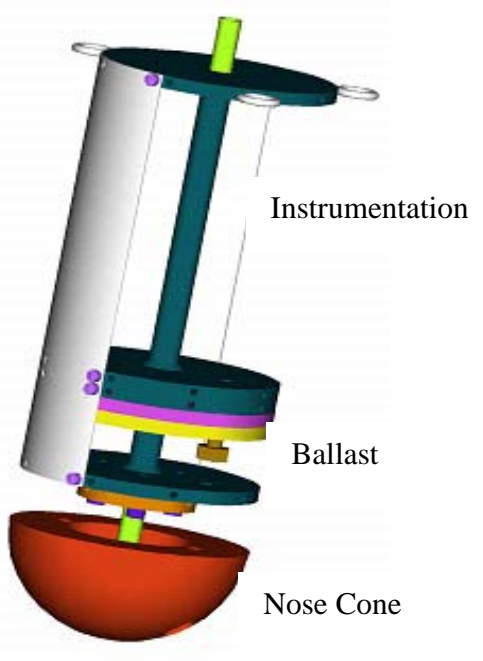

Figure 3. Parachute Payload.

The adjustable ballast weights provided the capability of evaluating the parachutes performance over a wider range of descent velocities. The payload instrumentation package incorporated a combination of custom designed components and commercial-off-the-shelf (COTS) hardware.

The camera system integrated into the payload for shape measurement included two programmable CCD cameras with 720x492 pixel resolution. The cameras were externally synchronized and programmed for a fixed exposure. The images were recorded to flash memory with the support of a miniaturized video recorder. High intensity LED's were included in the design to support the on board cameras. The analog data including the accelerometer, load cells and velocity probe were all acquired through a miniature 16 channel acquisition card. The power for all the components was provided by a custom lithium-ion battery, regulated through a custom power distribution card while power requirements ranged from 5 to 24 volts. The payload instrumentation was remotely operated through a 6 channel radio frequency (RF) controller. A detailed description of each sub-system component is described below.

\section{A. Cameras}

Three on-board video cameras were provided to support both qualitative and quantitative aspects of the deployment and descent during testing. Two of the cameras were configured to exclusively support the photogrametric measurement and the third was provided to record surveillance video of the descent. Each of the three cameras was monochrome $(\mathrm{B} \& W)$. The cameras operated at a $30 \mathrm{~Hz}$. frame rate and provided a standard analog video output signal conforming to National Television Standards Committee (NTSC) specifications. The standard output signal in this format is interlaced. The interlaced video is composed of 2 fields that are captured at different moments in time. ${ }^{4}$ The time interval between the generation of the 2 fields is $1 / 60^{\text {th }}$ of a second. This small delay will exhibit motion artifacts when both fields are combined and displayed as a static image. To 
overcome this characteristic in our application the NTSC video requires special processing to deal with motion of the canopy. An example of the processing technique is described in more detail later in this report.

The miniature cameras provided a programmable interface utility to control exposure and gain, critical features in recording an object in motion, like the parachute, to prevent the adverse effects of motion artifacts. The synchronization of the video cameras in a photogrammetric measurement system is the most critical issue. To support this requirement, the cameras have an option of external synchronization.

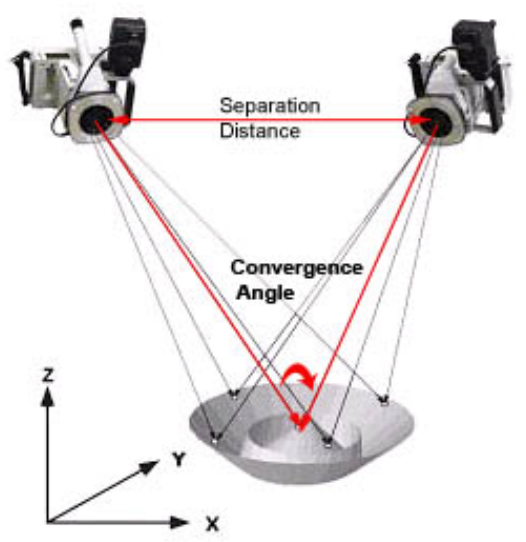

Figure 4. Photogrammetric camera-target geometrical relationship (typical).

The photogrammetric measurement technique can be described as a geometric solution of the intersecting light rays received by the cameras from an object "point", referred to in the context of this report as a target. The diagram in Figure 4 shows the typical geometric relationship between the point(s) to be measured and the camera positions. It can be shown that the separation distance between the cameras and the corresponding convergence angle are linked to the precision which the target point can be measured. ${ }^{5}$

Simulation and modeling were used to identify the minimum separation distance needed between the two arm-extended onboard cameras. Simulation indicated a minimum separation distance of 18 inches is needed for the payload cameras to achieve the desired precision of 0.01 inches in the XY plane. Two camera support arms extending from the payload provided the means to achieve the needed separation.

An adjustable camera mounting bracket supported by a rod extended from the payload was designed to provide rotational and translational freedom in 2 axes. The camera mounted on the support arm is shown in Figure 5.

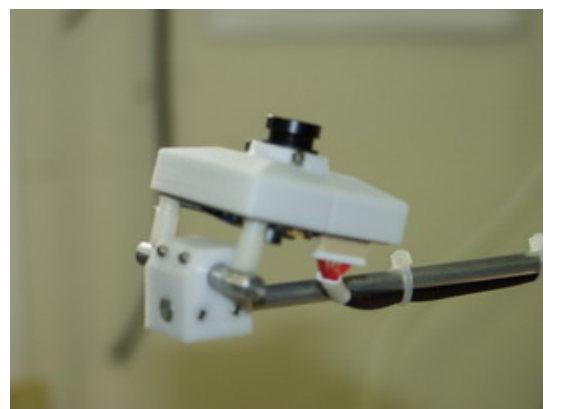

\section{B. A/D board}

An embedded microprocessor with an 8 channel 16-bit Analog to Digital (A/D) converter was incorporated into the package to support the acquisition of the various analog sensors. The acquisition sequence was synchronized to the video acquisition process through an external trigger. Signal conditioning for each channel was provided through a custom design.

Figure 5. Payload Camera/Mount.

\section{Video Recorder}

Single channel video recording was made through a miniature digital video recorder (DVR) circuit board. An individual recorder was required for each camera. The DVR, provided a high quality digital video recording and playback device designed for stand-alone operation and rugged applications. A constraining factor was the unit's limited compatibility with video conforming to NTSC specifications only. A requirement of future applications will be a recorder compatible with progressive scan formats. The video recorder performed compression conforming to the Joint Photographic Experts Group (JPEG) standard at a specified 4:1 at 30 frames per second. The DVR provided a triggered input to initiate recording, this feature provided the means to insure the video sequences from each camera were simultaneously recorded. The trigger was initiated remotely through RF control. When recording, the DVR converted an analog NTSC video signal to a component digital format, compressed the digital video signal to a JPEG data stream, and sent the compressed video directly to an on-board compact flash memory card. The flash memory cards used during these tests provided the means to record more than 4 minutes of video. After each drop the video files were downloaded to a computer via USB port for processing. The individual DVR circuit board and the payload installed version are shown in Figure 6. 


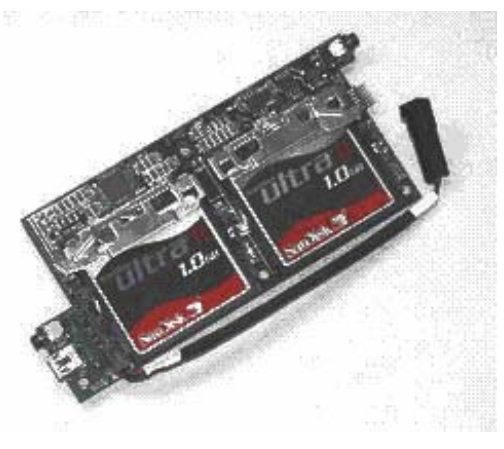

Figure 6a. Digital video recorder (DVR)

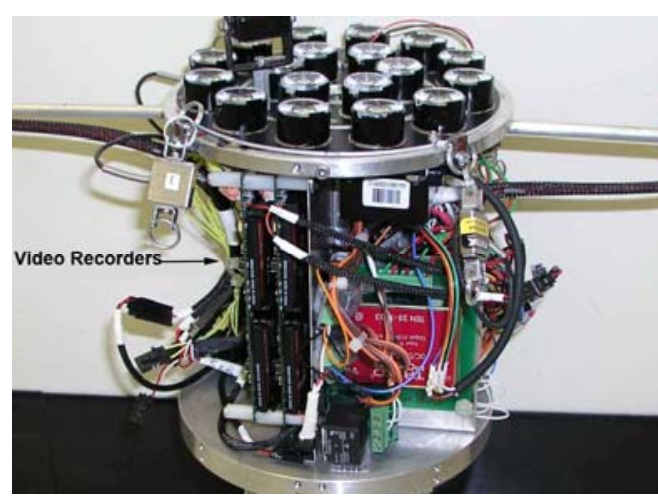

Figure 6b. Payload installed DVR's

\section{Power Management}

The power requirements for the various sub-system components in the payload required the design of a distributed low voltage dc system. The battery was assembled from lithium ion cells to provide 14.4 vdc at 1500 mAh. The battery power was regulated and distributed through a custom built module. The power module included a radio frequency (RF) controlled relay to provide on/off control and minimize battery drain during the predrop operations.

\section{E. Lighting}

The on-board lighting played a critical role in the measurement of the canopies shape in addition to a secondary role for visible illumination. The photogrammetric shape measurement technique requires three basic components; the camera, lighting and targets. The fidelity of the shape solution is directly linked to the target density and the corresponding point cloud. The targeted points for this application are made with adhesive backed retroreflective material. To achieve maximum signal return from the retroreflective target the cameras and the light source need to be concentric. The retroreflective signal provides a well defined contrast in the imagery between the target and the associated background. For the payload lighting system, a 24 volt high-intensity multi-LED array was designed. The LED's were visible white light and utilized a non-standard lens to project a $52^{\circ}$ divergence cone to fully encompass the canopy in its fully deployed shape. The LED lighting system is shown in Figure 7. The LED’s were independently radio controlled (RC) to economizing the power drain on the battery. This feature allowed the lighting on/off operation to serve as a synchronization check for the recorded video from the on-board cameras.

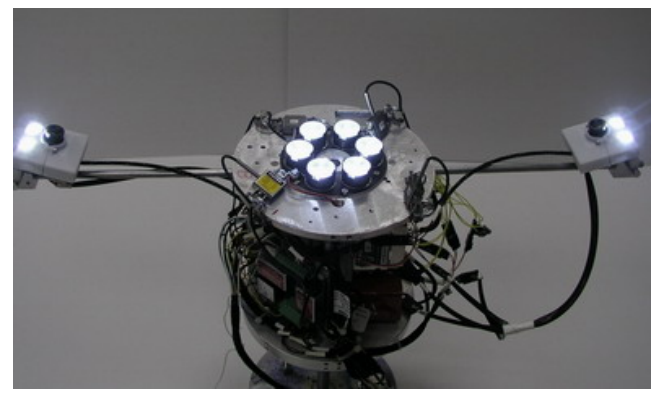

Figure 7. High Intensity on-board LED lighting.

\section{F. Velocity}

With the payload drop constrained in the z-axis the ability to measure total velocity or descent rate was achieved with an on-board probe. The probe uses a sensing technique known as hot wire anemometry. A very fine wire (on the order of several micrometers) is heated to some temperature above ambient. The air flow past the wire during the parachutes descent has a cooling effect on the wire and alters its electrical resistance. The change in resistance is monitored as a change in voltage and recorded as part of the analog to digital acquisition. 


\section{G. Accelerometer:}

The package included a commercially available Tri-axial accelerometer with a $\pm 10 \mathrm{~g}$ range. The unit incorporates a Micro-Electro-Mechanical Systems (MEMS) sensing element that provides good noise immunity and stability. The unit's analog output range of $5 \mathrm{Vdc}$ required a simple voltage divider signal conditioning circuit to provide a compatible signal level with the analog to digital converters $2.5 \mathrm{Vdc}$ range. The unit was independently calibrated pre and post test to validate its performance and the integrity of its signal.

\section{H. Load Cells}

Four strain gauge load cells provided the coupling between the canopy's suspension lines and the payload. The load cells provided high precision tension load measurement over a $50 \mathrm{lb}$ range. The installed load cells are visible in Figure 8.

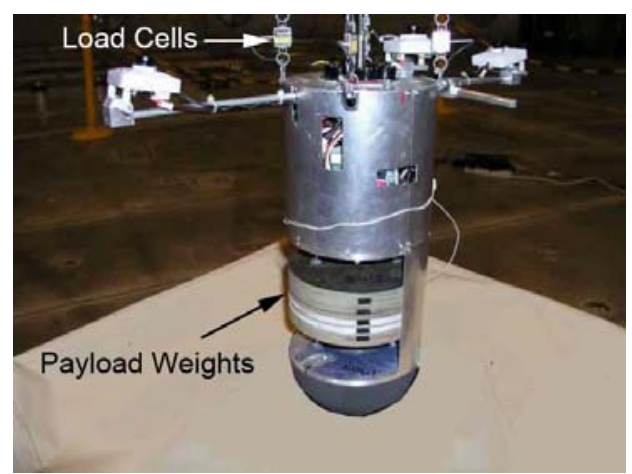

\section{Payload Weights}

The fully instrumented 6 inch payload package weighed 6.705 lbs. To achieve a wider range of payload velocities a variety of machined aluminum and steel plates were produced to serve as ballast weights. The stackable weights were easily installed with the removal of the payload cover plates. The plates were secured in the package by threaded alignment rods. The view of the payload in Figure 8 shows the covers removed and 10 lbs. of ballast installed.

Figure 8. Payload ballast and load cells.

\section{Shape Measurement}

\section{A. Coordinate System}

The coordinate system for a photogrammetric measurement would normally default to a reference based on the position of the cameras unless another is provided through an external reference. For our testing it was desired to have the z-axis parallel the guide wire. To establish this "guide wire" reference, an independent targeted frame was built to serve as a reference for the intersection of the XY axis. The frame, shown in Figure 9a., was lifted along the guide wire into a position 80 feet off the chamber floor. Once positioned a "constellation" of points were added to the crane structure that were linked to this new coordinate system. A view of the crane and the additional retroreflective reference points is shown in Figure 9b. These new fixed points provided a reference to the desired coordinate system for target points measured on the canopy during its descent.

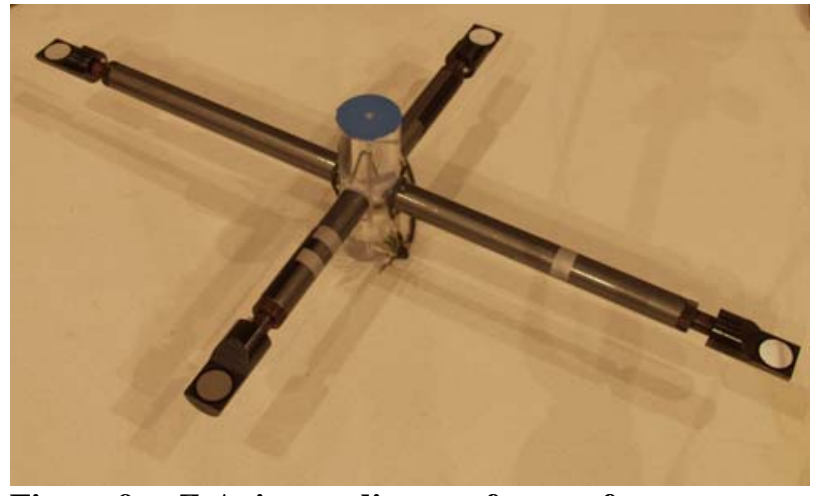

Figure 9a. Z-Axis coordinate reference frame.

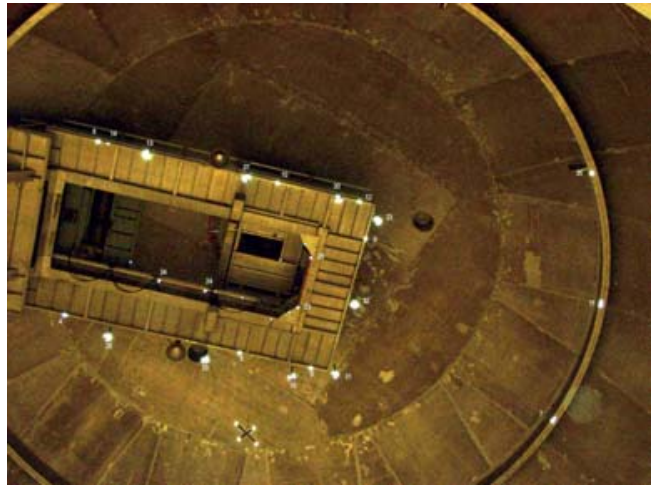

Figure 9b. Retroreflective targets added to crane

\section{B. Canopy: Photogrammetric Target Configurations}


The frame rate and resolution of the video cameras must be considered when designing a target pattern for shape measurement of the canopy. The testing revealed the limitation in how dense a target pattern could be with the instrumentation and cameras that were chosen. An example of a target pattern tested whose targets were too closely spaced is shown in Figure 10a. A closer inspection of a sequence of images in the video revealed the frame-toframe motion of most targets to be greater than the original separation distance in the image. To accurately track a particular target over a sequence of images such as this would require a more robust algorithm capable of tracking the targets in both image space coordinates (2D) and object space coordinates (3D) than is currently available. However, the utility as just described is currently under development and should be available to support future test and follow-up analysis of the data collected as part of these ground tests. Alternatively, this problem can be avoided with higher frame rate cameras resulting in smaller steps across the image or reducing the target point density to a level where the motion does not create an ambiguity. Examples of the target configurations used on the $7.5 \mathrm{ft}$ canopies are shown in Figure 10a-c. The images were taken with ground-based cameras.

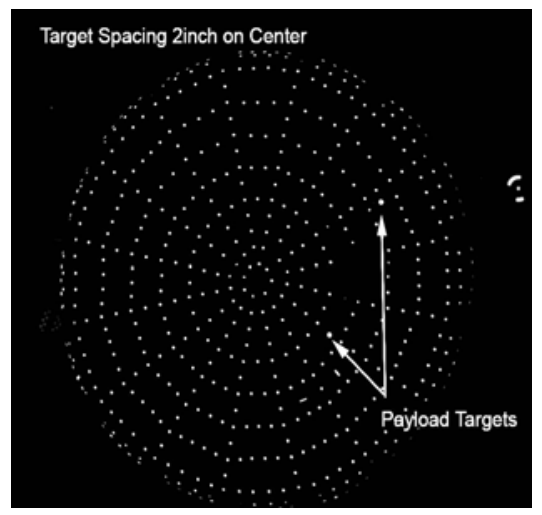

Figure 10a. Spatially dense target pattern. Early test configuration

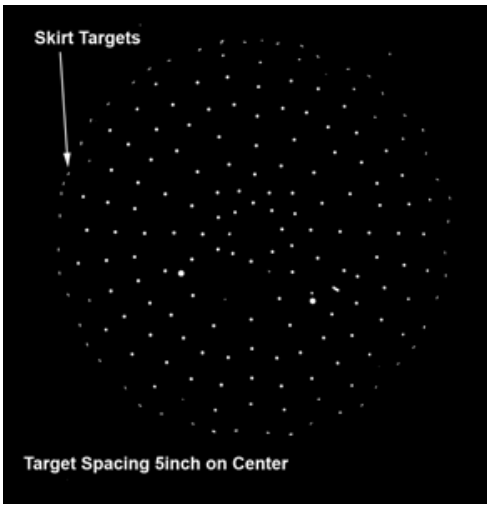

Figure 10b. Target pattern (typical) used for most drop test.

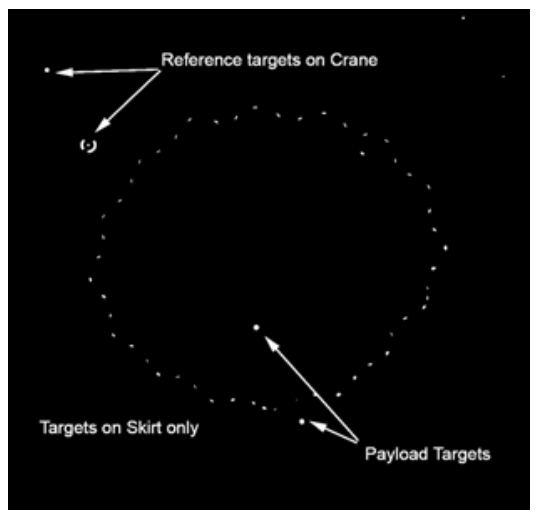

Figure 10c. Canopy with skirt targets only.

\section{Image Processing: De-Interlacing}

The ability of the photogrammetic measurement technique to produce highly accurate shape measurements is linked to its ability to precisely measure the centroid of circular targets used to describe the shape of the object. In this application the object's shape of interest is the canopy. As previously discussed, the on-board video cameras record information in a NTSC format. The image produced from the interlacing of the two fields often yields a false centroid when the effects of motion are not considered. To resolve this undesirable effect the image can be "deinterlaced" and processed as individual fields. There are pros and cons to this effort, the splitting or de-interlacing of the image removes the motion artifacts visible in the composite image and provides an effective frame rate of 60 $\mathrm{Hz}$, but it also cuts the vertical resolution in half typically resulting in higher overall error. The image shown in Figure 11 highlights the effect of motion on the recorded image when viewed as a composite of the two fields. The corresponding de-interlaced image(s) in Figure 11b describe the target's true position on the image plane at that point along the drop. 


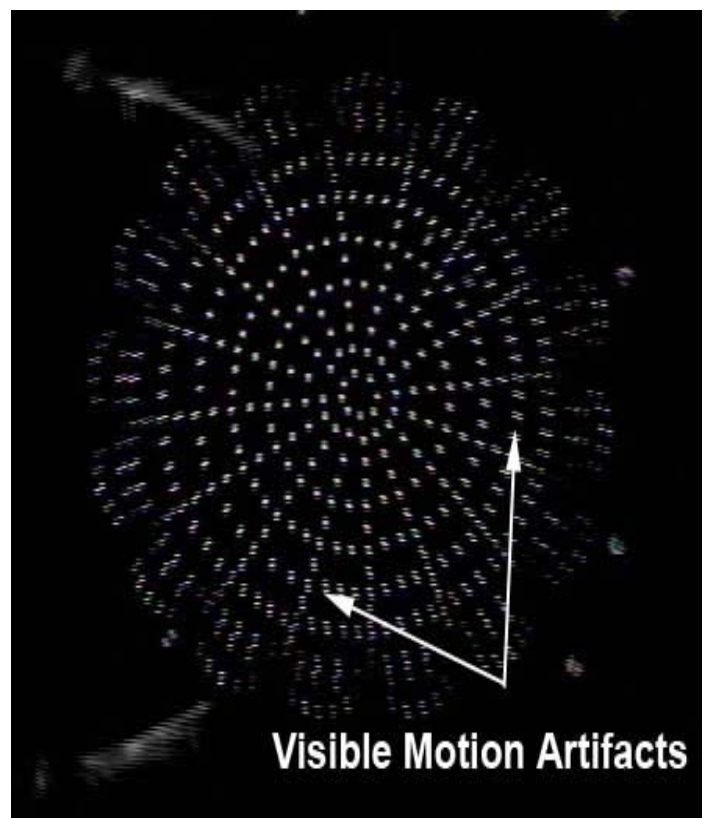

Figure 11a. Composite image with visible motion artifacts from interlacing.

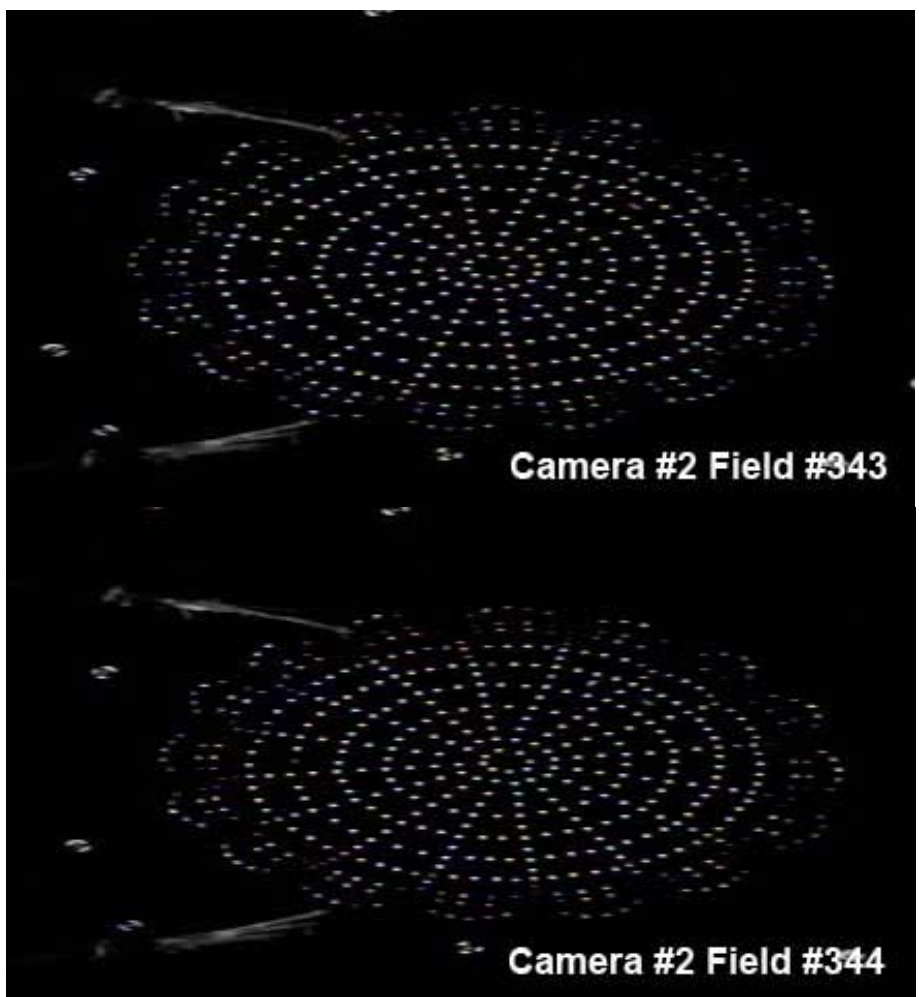

Figure 11b. De-interlace image, without motion artifacts

\section{Testing}

Test drops were conducted in sets of 10 for a particular payload weight. The initial set was performed with only the base payload weight of $6.7 \mathrm{lbs}$, subsequent sets were performed with weight increments of 5, and $10 \mathrm{lbs}$. Payload velocities at the $10 \mathrm{lb}$ load exceed $23 \mathrm{ft} / \mathrm{sec}$ and the $10 \mathrm{~g}$ shock load exceeded the limit of most of the components within the package. A typical example of the canopy's shape derived from the photogrammetric solution is shown in Figure 12. The example reflects the change in shape of the canopy's skirt over a one second period during the descent. The vectors connecting the points represent the tracked path of a particular point through the sequence of video images. A detailed discussion of the test results will be provided in future reports. 


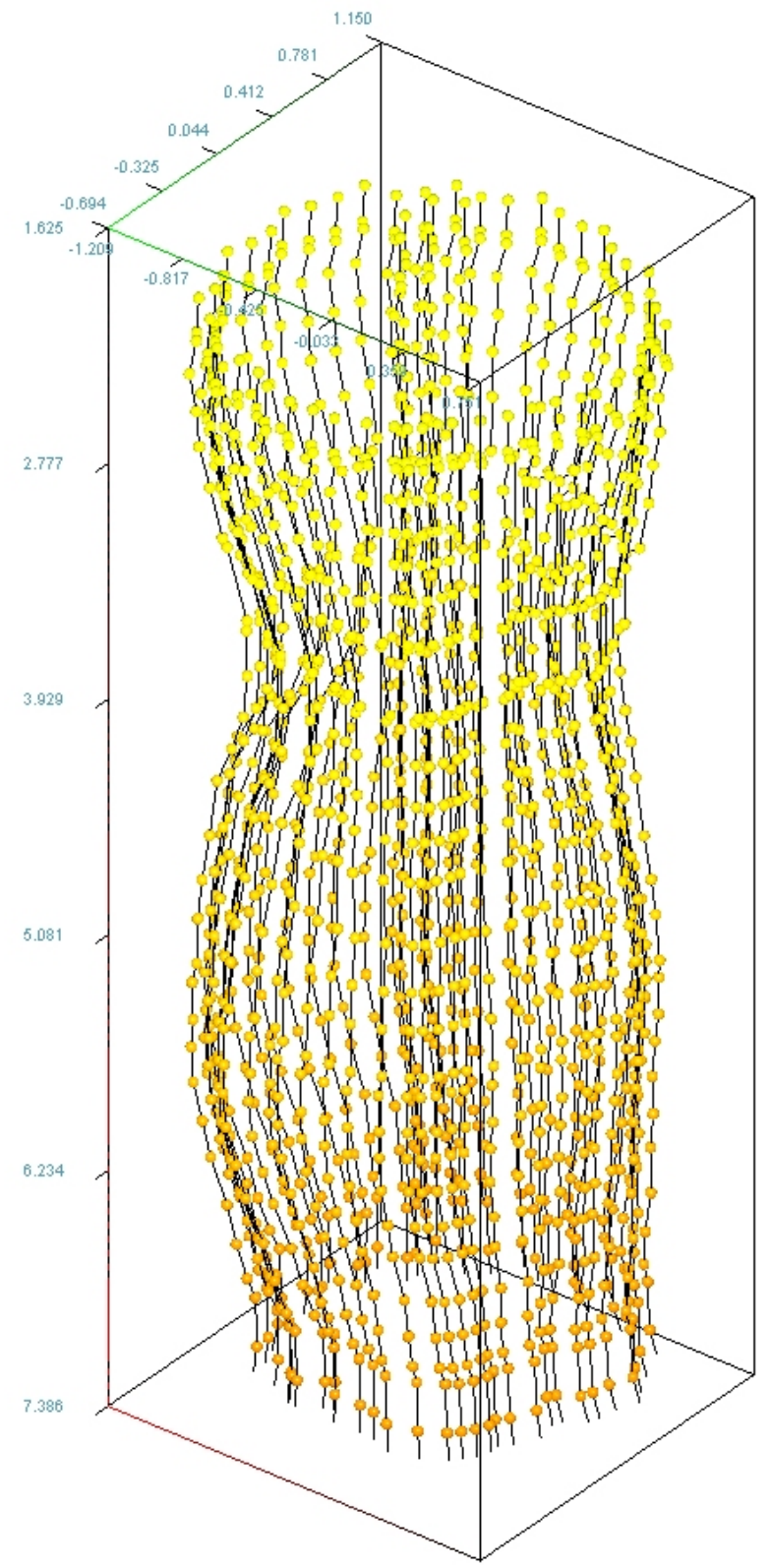

Figure 12. Canopy's skirt shape measurement data (typical) recorded over one second interval.

\section{Conclusions}

This report discussed the design and development of an instrumentation package for experimental testing of scaled circular parachutes. The tests were conducted in an environmentally controlled chamber from a drop point 90 feet above the floor. The payload package incorporated instrumentation for shape, acceleration, load, and velocity measurements and operated under RF control. The package performed well during ground test and proved capable of measuring and surviving the physical parameters involved during the inflation and decent phase of parachute air drop. The effort provided quantitative shape information as well for both the canopy and the skirt. The photogrammetric methods require careful analysis of the expected motion as it relates to the physical separation distance between adjacent targets. Target patterns where the separation distance as seen by the cameras is small 
may result in ambiguities in target tracking that cannot be easily resolved. Design specifications are currently being reviewed for development of a similar package capable of supporting larger scale open air parachute drops from altitudes up to $3000 \mathrm{ft}$.

\section{References}

${ }^{1}$ Heinrich, H.G. and Noreen, R.A., "Analysis of Parachute Opening Dynamics with Supporting Wind Tunnel Experiments," AIAA $2^{\text {nd }}$ Aerodynamic Deceleration Systems Conference, El Centro, California, Sept. 2325, 1968, AIAA Paper 68-924.

2 Knacke, T. W., Parachute Recovery Systems Design Manual, $1^{\text {st }}$ ed., Para Publishing, Santa Barabara, CA, 1992, Chap. 5, 91-96.

${ }^{3}$ Meyer, C. G., Jones, T. W., Lunsford, C. B., and Pappa, R. S., "In-Vacuum Photogrammetry of a 10-Meter Solar Sail,” 46th AIAA ASME ASCE AHS ASC Structures, Structural Dynamics and Materials Conference; Austin, TX; 2005.

${ }^{4}$ Shortis, M. R. and Snow, W. L., "Calibration of CCD cameras for field and frame capture modes," Conference on Digital Photogrammetry and Remote Sensing '95, SPIE Vol. 2646, pp. 2-14 (1995)

${ }^{5}$ Cooper, M.A.R and Robson, S., "Theory of close range photogrammetry," Close Range Photogrammetry and Machine Vision, edited by K.B. Atkinson, Whittle Publishing, Scotland, UK, 2001, pp 9-50. 